\title{
Effect of Geriatric Program on Achieving Sustainable Development Goals and Universal Health Coverage among Older Persons in Africa; A Nigeria Project Plan
}

\section{Amara Frances Chizoba MPH PhD ${ }^{1 *}$,Chukwuebuka Ugwu MPH, MBBS $^{1,2}$ and Chinedu Asiegbu MD ${ }^{1,3}$}

${ }^{1}$ Mission to Elderlies Project Department, Renewal Health Foundation, Nigeria

${ }^{2}$ Public Health Department, World Health Organization, Nigeria

${ }^{3}$ Epidemiology Department, Sam Houston State University, USA

*Corresponding Author: Amara Frances Chizoba, Renewal Health Foundation,

Nigeria. E-mail: missiontoelderlies@gmail.com, info@renewalhealthfoundation.org

Phone: +2347088698103; Website: https://www.renewalhealthfoundation.org/
Received: July 01, 2021

Published: November 15, 2021

(C) All rights are reserved by Amara Frances Chizoba., et al.

\begin{abstract}
Introduction: Population of older person are growing globally, in Africa and Nigeria. The WHO stated that this demographic change has implications on health care systems and on achieving sustainable development goals (SDGs) and universal health coverage (UHC). However, programs to adjust to population aging is still lacking in countries like Nigeria.

Aim: This project proposal aims to implement and evaluate, with goal of scaling up, community geriatric program on achieving SDGs and UHC among older persons in Nigeria.

Methodology: Intervention approach will include home based management of older persons through seven geriatric case management processes, service provisions by trained case managers across four intervention domains, instituting and supporting health geriatric clinics and drug banks, enrollment of beneficiaries into national health insurance program and supporting system strengthening (on geriatric care) for service providers and hospitals. Data collection and entry will be done using expert data capturing tools and geriatric data management information system (GDMIS), while data analysis will be done through comparison of pre and post intervention indices.

Result, Conclusion and Recommendation: Review of literatures and anecdotal data show paucity of geriatric projects for care of older persons whose population is exponentially increasing in Africa. This project proposal that aims to implement and evaluate effect of a novel and innovative community health programs on achieving SDGs and UHC goals among older persons in Nigeria will share its result, conclusion and recommendations, which will be disseminated for adaption and adoption for geriatric programing in the African context.
\end{abstract}

Keywords: Older Persons; Health Program; Universal Health Coverage; Sustainable Development Goal; Nigeria

\section{Introduction}

Demography of aging and population aging

The World Health Organization (WHO) defines older persons as those aged 65 years and older. Today, population aging- a phenom- enon used to describe an increasing median age in a population due to declining fertility rates and rising life expectancy- is a strong discussion point as most people are living longer and a significant proportion of the world's population are getting older. According to World Health Organization [1], by 2020, the number of people aged 
60 years and older will outnumber children younger than 5 years and between 2015 and 2050, the proportion of the world's population over 60 years will nearly double from $12 \%$ to $22 \%$ [1]. The United Nations [2] in 2017 reported that over the coming decades, the number of older persons is expected to grow fastest in Africa, where the population aged 60 or over is projected to increase more than threefold between 2017 and 2050, from 69 to 226 million. Population of older person are therefore growing exponentially globally, and Africa is reported to experience the fastest growth in the coming decade [3]. Whereas in Sub-Saharan Africa, it is estimated that the number of elderly persons will rise from about $37.1 \mathrm{mil}$ lion in 2005 to 155.4 million in 2050 [4,5]. In 2015, 4.5 per cent of Nigeria's population was aged 60 years or over and that proportion is projected to increase by 4.8 per cent through 2030 [5]. But by 2050 , however, the proportion of older persons in Nigeria is expected to have begun to grow more significantly, reaching 6.3 per cent in the medium variant projection [5]. According to Africa Central Intelligence Agency (CIA) [6], Nigeria has several older persons with $5.1 \%$ elderly dependency. Thus, out of the large population of 203,452,505 (July 2018 est.) [6], the absolute number of older persons that form the dependency ratio is relatively high. Nigeria, population of older persons aged 60 years and over are $3.26 \%$ of the population; 3,138,206 males and 3,494,524 females (with total of 6632730) [5-7]. This demographic change has strong implications for goals of sustainable development and universal health coverage. Thus, supporting these changes by taking multi-sectoral action on ageing and health is very critical.

\section{Healthy aging and aging in place}

Interwoven with population aging phenomenon are concepts of healthy aging and aging in place. Healthy Ageing is defined by the World report on ageing and health as the process of developing and maintaining the functional ability that enables well-being in older age. The health issues that confront older people are often associated with chronic conditions that can be prevented or delayed by engaging in healthy behaviors across the life course. Other health conditions can also be effectively managed, especially if they are detected and treated early. And for older adults with significant declines in capacity, supportive environments and integrated care systems can ensure that they live lives of dignity and continued personal growth [8]. On the other hand, is another important concept in care of older person known as aging in place. Aging in place refers to an older adult's ability to live within their own homes and communities, rather than relocating in later life, especially for reasons such as functional decline, widowhood, or financial constraints [9]. A goal of aging-in-place, therefore, can be framed as optimizing the interaction between older adults and their environments, including their living with their family, loved ones and community. In African culture like in Nigeria, interventions to promote healthy aging while encouraging aging-in-placewhere older persons live in their homes and familiar communities as opposed to old people home is seen as being culturally sensitive. This concept is seen to respect the culture of younger ones taking care of the older ones which is believed to be a blessing in Africa. Therefore, any health intervention that incorporates services closer to the homes and communities of the older persons is expected to receive more acceptability and accessibility by the older persons and their families.

\section{Challenges of population aging}

Challenges of the current rapid population aging are quite enormous. According to WHO [1] the challenges ranges from increasing need for health care access to increasing health expenditures/cost, increasing use of health facilities and increasing need for trained health care workers on health care of older persons. As impact of population aging increases on health care system, the need to provide interventions that meets the challenges cannot be overemphasized. These interventions should be aimed to achieve universal health coverage and sustainable development goals especially as it relates to older persons.

Universal health coverage, Sustainable developmental goals and Aging

Universal Health Coverage (UHC) is defined by WHO as ensuring that all people and communities receive the quality services they need, and are protected from health threats, without financial hardship [10].

Therefore, this means that the world health organization's definition of UHC suggests that UHC ought to enable everyone to access the services that address the most significant causes of disease and death and ensures that the quality of those services is good enough to improve the health of the people who receive them. And protecting people from the financial consequences of paying for health services out of their own pockets reduces the risk that people will be pushed into poverty because unexpected illness requires them 
to use up their life savings, sell assets, or borrow. Despite the need for UHC, it has been reported that achieving UHC has been poor, especially in poor lower- and middle-income countries like Africa.

Population ageing will have an impact on the ambition of universal health coverage, because without considering the health and social care needs of the ever-increasing number of older people, UHC will be impossible to achieve. Equally, Sustainable Development Goals (SDG) especially SDG 3 - "ensuring healthy lives and promoting well-being for all at all ages" - [1] cannot be met without transforming health and social systems from a focus only on disease towards the provision of the integrated and person-centered care that is known to have the greatest impact on functional ability in older age.

This Transformation requires specific attention to the following: [10] organizing services to respond to older people's diverse levels of capacity as well as their needs and preferences; extending coverage of services to all older people - at present many older people, particularly those who have been in the informal workforce or who have filled caregiving roles, have very limited access to even basic services including drugs; ensuring that coverage extends to services that provide interventions that are key for maintaining intrinsic capacity and functional ability of older people (for example comprehensive assessment and care plans, provision of medications especially for the chronically restorative surgery, assistive devices, functional nutritional supplements, multimodal physical exercises and long term care); developing sustainable financing mechanisms that can protect older people and their families from undue financial burdens and that provide system incentives for the provision of the services older people need.

By adopting affordable, integrated and person-centered service delivery models and comprehensive systems of long-term care, based in the communities where older people live, we can accelerate progress towards UHC, and Sustainable Development Goal [10]. Ritu S., et al. [11] in their document titled "WHO, universal health coverage must include older people", stated that although every country is different, steps can be taken towards UHC and SDGs achievement strategies that are more inclusive of older adults. Thus, countries need to foster better integration between health and social care to improve and maintain older adults' physical and cognitive capacities. Example of which include provision of health and health related services, psychosocial and economic support services to older persons in their familiar community and possibly by their indigenous community health workers. Araujo de Carvalho I., et al. [12] in their study reported that older people who benefit from coordinated chronic care that is guided by comprehensive assessments report more satisfaction and experience fewer emergency referrals than when they receive services that treat individual conditions independently. In order words, older persons with chronic conditions may benefit better from a coordinated community-based intervention, which covers a wide range of older persons needing similar chronic care than mere individualized hospital-based services for probably a few that can access it. This approach of community-based intervention is aimed to encourage aging in place while implementing interventions for healthy aging. To achieve community-based intervention proposed by the authors, community health worker needs to be trained and empowered to provide health care services to older persons in their homes. Carvalho I., et al. [12] further explains that efforts to ensure UHC and SDG are achieved especially among older persons should involve policymakers who would negotiate ways to reduce costs and increase risk sharing, probably bringing into their discussion a strong public-policy partnership. Such partnership could be in form of partnership of governments with pharmaceutical companies to share the cost of making life saving medication available, accessible, and mostly affordable for the chronically ill older persons-who usually have comorbidity and need for polypharmacy- through primary health centers and community pharmacies.

Health status, comorbidity and polypharmacy among older persons

Older persons are faced with chronic conditions, thus plan to make medications accessible and affordable to them will aim to reduce out of pocket expense which has a ripple effect in achieving UHC. Usually, older adults have multiple medical problems. Almost $50 \%$ of the elderly population has at least three chronic diseases, and of this, approximately $10 \%$ has five or more [13]. These diseases can coexist for several reasons such as by random chance because they are part of the same continuum, because they have risk factors in common or because one triggered the other. Aging and comorbidities together increase the risk of hospitalization and mortality. According to a study reported by Christine TK., et al. [14], people aged seventy-five and older report an average of three chronic health problems at any time and use more than 4.5 prescription drugs. Their study revealed that among the chronic 
conditions reported by noninstitutionalized older adults are arthritis (56 percent), hypertension (55 percent), heart disease (32 percent), and urinary incontinence (17 percent). In addition, the incidence of many other illnesses, such as many cancers, osteoarthritis, and hip fractures, also rises with age, thus S. L. Rosen and D. B. Reuben in their book titled "Geriatric Assessment tools" [15] listed common medical/health conditions among older persons for which assessment and intervention must be planned.

Common medical/health conditions among older persons for which assessment and intervention must be planned [15]

- Falls/Gait Disturbance: More than one-third of community-dwelling older persons fall each year, and falls are independently associated with functional decline. Also, patients who have fallen are at high risk for falling again and having resulting injuries. Because older persons frequently attribute falls to normal aging, it is very important to ask older patients if they have fallen in the last year, at their initial visit and at least annually. The use of a pre-visit questionnaire can help elicit this information efficiently.

- Hearing Impairment: Hearing impairment affects up to one-third of persons aged $>65$ years. Independently, it is associated with reduced cognitive and physical function, and reduced social involvement. It is also often under-recognized and therefore undertreated, and again often not self-reported by patients. There are several methods to help screen for hearing loss. One simple method for a busy practitioner is simply to rely on the patient's own subjective report of hearing loss.

- Visual Impairment: Visual impairment is a common sensory deficit in the older population; all 4 major eye diseases (cataracts, macular degeneration, diabetic retinopathy, and glaucoma) increase in prevalence with age. Most older persons have presbyopia and require corrective lenses. Visual deficiencies are also independently associated with increased risk of falling, functional decline, and depression.

- Urinary Incontinence: Urinary incontinence is under-reported as well, often due to patients' embarrassment or belief that incontinence is a normal part of aging. In fact, it is a very common problem in both older men and women, and can have deleterious effects on their lives, including urinary tract infections, sleep disruption with subsequent falls, and pressure ulcers. It is also a marker for higher mortality in older adults. There are many treatment options available, including behavioral, pharmacologic, and surgical.

- Malnutrition/Weight Loss: The term "malnutrition" has been used to refer to a wide spectrum of deficiencies (eg, proteinenergy, vitamins) and excesses (eg, obesity, hypervitaminosis) that place older persons at risk for other health conditions, functional decline, and death. Nutritional disorders are very common in older persons, the most common one being obesity

- Polypharmacy: Adverse drug effects have been a challenge with orthodox medicine. For instance, some hypertensive drugs are associated with dizziness and headache. Much more in older person who take a handful of medicine due to comorbidities. Polypharmacy is very common in the older population, associated with adverse drug reactions, and can result in hospitalizations and increased morbidity. Patients often visit $>1$ healthcare provider and fill prescriptions at multiple pharmacies. Therefore, monitoring the many medications patients are on for drug-disease and drug-drug interactions is imperative at the initial and every follow-up visit.

- Other conditions: Include issues related to psychoscocial and economic domain that affect health status of older persons. Such as mental health (cognition, delirium, dementia etc), social isolation and financial dependency due to retirement and inconsistent pension scheme of nations.

Unfortunately, the aging and comorbidities that come together are usually chronic in nature, and comorbid chronic illnesses come with need for polypharmacy. Multiple medication prescription to manage the chronic comorbidity among older patients can therefore result to increase in health cost especially out of pocket expenses for majority of the older persons who may not be insured in African countries like Nigeria. And as such, interventions to ensure medication are available for older patients are crucial to ensure they don't fall into financial hardship thus falling short of goals of UHC.

Access to Health care and medication among older persons

On assessing access to medication by older patients, WHO [16], estimated that two billion people have no access to essential medicines, effectively shutting them off from the benefits of advances in 
modern science and medicine. Lack of access to medicines is one of the most complex - and vexing - problems that stand in the way of better health. Affordability is the cornerstone of access. And if so, intertwine of chronicity, co-morbidity, polypharmacy, retirement (resulting to reduction in come) among older persons have a compound effect on their affordability of medication. A 2019, a US publication identified that prescription drugs are a major component of the overall cost of caring for the elderly [17]. That persons sixty-five and older spend an average of over three percent of their income on prescription drugs. And that percentage is even higher when over-the-counter medications are included. Also, the publication reported that over sixty-five percent of seniors' prescription costs are out-of-pocket. They further suggested three ways by which older persons can better afford the cost of prescription drugs as thus; health insurance coverage; financial assistance that helps pay for medication such as pharmaceutical company assistance; and non-governmental organizational assistance (such as Needymed who provides drug subsidy for up to $80 \%$ of prescription drug for older patients in US).

\section{Concept of drug bank or drug subsidy for older persons}

Geriatric Drug bank or drug subsidy for seniors are terms used to describe programs adopted by countries to make prescription drugs available to older persons at a low or affordable costs [17]. The programs provide drugs to older persons through pharmaceutical companies at subsidized costs, social protection like health insurance or financial assistance like tax deductibles or direct cash transfer especially in cases of NGOs. Example is the Elderly Pharmaceutical Insurance Coverage (EPIC) program- a New York State program for seniors administered by the Department of Health that helps more than 337,000 income-eligible seniors aged 65 and older to supplement their out-of-pocket drug plan costs [18]. Although some of these plans and programs exist in developed countries, plans or programs to assist older persons' medication affordability and accessibility are not readily available in developing countries like Nigeria. And where available as in the case of national health insurance program, older persons are either not listed to benefit as one of the vulnerable groups or their chroniccondition- related- medications are not fully covered by the health insurance plan. Thus, such medication programs must be explored to help reduce out of pocket cost of medication for older persons in order to improve their access to their life saving drugs.
WHO has further explained that many other factors also determine whether people get the medicines they need. For instance, gaps in local health systems and infrastructures hamper the delivery of medicines to millions of people. Access also depends on procurement practices, tax and tariff policies, mark-ups along the supply chain, and the strength of national drug regulatory authorities. These numerous challenges of access to quality medicine comes to impound on already existing strain of achieving universal health coverage among the older persons [16]. For instance, a retired sixty-five-year-old woman that has comorbidity and prescribed polypharmacy who does not have a health insurance coverage will need a support with accessing medication or stand the chance of missing lifesaving medicines. A study done in Nigeria by Chizoba AF, et al. [19] revealed that only $22 \%$ of 521 chronically ill older patients assessed could afford their medication, whereas $78 \%$ reported to have missed medications owing to unaffordability/cost. On the other hand, only $18 \%$ are enrolled into health insurance program to aid health and medication access. The authors thus concluded that chronically ill older patients in Nigeria cannot always afford medications and majority are not enrolled into health insurance program to aid health and medication access and thus, aim of UHC is short of being achieved among the elderly in Nigeria. They further recommended that innovative strategies - such as enrollment of elderly persons into health insurance program to aid access to health care, improving access to medication and providing socioeconomic support for the elderly - should be implemented to improve UHC among the elderly in Nigeria. However, aside tackling cost of medication for older persons, there is need to take into consideration its accessibility. To make medication or health care services in general accessible, bringing the services closer to the community of the target population is important. And since WHO encourages aging in place, it is important to consider bringing the medications closer to the community where majority of the older persons live and/or retire into.

Community based approach to increase health and medication access among older persons

Community health programs have been found to be effective. In their study, Eugenia LS., et al. [20] explained that Communitybased supports and services (CBSS) are designed to help community-dwelling older adults remain safely in their homes and delay or prevent institutionalization. CBSS provide (and act as a link to) 
specific resources for older adults and their caregivers that include wellness programs, nutritional support, educational programs about health and aging, and counseling services for caregivers, as well as general assistance with housing, finances, and home safety. With the rapid aging of population, even as overall health improves the number of older adults who could benefit from CBSS is expected to increase significantly in the coming years [20].

One of the benefits of community health program is that it is implemented by community members trained as health workers who live and work in their communities thus increasing health workforce and access to services. Example of such successfully implemented community health workers driven projects in Nigeria is the HIV program and orphan and vulnerable children (OVC) programs. In the HIV program, community members are trained as community health workers to deliver ARV medications and provide health related services such as adherence counselling to the patients in their community (through community adherence groups). On the other hands, in the OVC program, the community health workers provide services to the vulnerable children and their household ensuring that not only health services are provided but that socioeconomic factors that affects health - such as issues of safety and economic stability- are supported. This model can be refined to suit community health program for older persons in Nigeria.

\section{Conceptual framework}

Programs for older persons should adopt the community-based approach especially in African setting like Nigeria where a good number of the population live in rural community settings. Training community health workers on community-based geriatric care to deliver services like health status assessment and referral, administration of available prescribed drugs, nutrition support, psychosocial care, social protection from elderly abuse and economic strengthening for the elderly or their caregivers. This service can be delivered through community health programs, community pharmacies and home visit. Irrespective of approach used, comprehensive geriatric assessment (CGA) model should be in-cooperated into the intervention approach from assessment, problem listing, goal setting, planning, intervention, and review/evaluation. On the other hand, CGA recommended 8 assessment domains to be incorporated, which include; medical, functional, psychological, social, environmental, advance care planning, spiritual, sexual/intimacy. There approaches will ensure that all or at least most aspect of health of older persons are addressed.

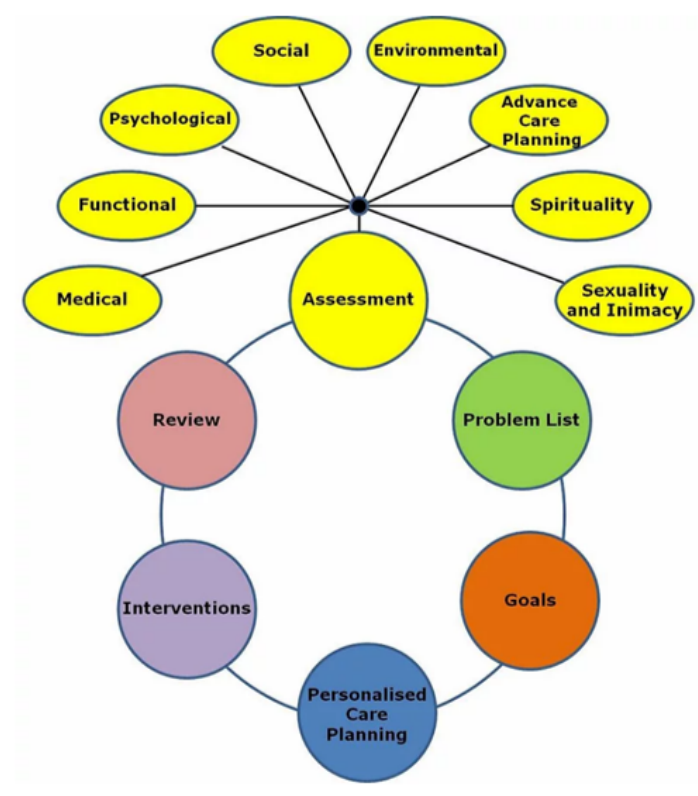

Figure 1: Conceptual framework for case management of community based health program among older persons.

\section{Purpose of Study}

Aim

To access effect of community health program on achieving SDGs and UHC goals among older persons in Nigeria.

\section{Objective}

- Describe health status and health care access among older persons in study group

- Institute and assess feasibility and functionality of community drug banks and community health program for older persons in pilot communities

- Evaluate effect of community drug bank on cost of health care and medications among participants before and after intervention

- Evaluate effect of geriatric community health program on health status and vulnerability index of participants before and after interventions. 


\section{Materials and Methods}

Study area

This pilot intervention study will be conducted in Eastern $\mathrm{Ni}$ geria. Nigeria is a country in West Africa with four regions (Northern, Southern, East and West). Each region constitutes of 5-14 states with eastern region having 5 states. Each of the states has relatively sparse population of older persons. Each state has local governments with communities. Within the communities are community and private health facilities including community pharmacies which serve the population with the older persons inclusive. This study will be conducted in three similar communities in three states (Imo, Anambra and Ebonyi States), in the eastern region of Nigeria with goal of scaling up subsequently to all parts of Nigeria.

\section{Study population}

The study population will comprise of older persons aged 60 years, resident in the study communities.

\section{Inclusion criteria}

The following will form the inclusion criteria;

- $\quad$ Older persons with reported age of 60 years.

- $\quad$ Older person clinically diagnosed or confirmed with at least one chronic health condition

- $\quad$ Resident within study area as at the time of study.

- Access to community health facility and community pharmacy.

\section{Exclusion criteria}

- Visitors within the study as at time of study.

- An older person unable to report his/her age to be 60 years

- $\quad$ Older person 60 years but without a clinically diagnosed or confirmed chronic health condition.

\section{Ethical consideration}

- $\quad$ Ethical clearance will be received from ethical committee in charge of the study communities

- Verbal and written consent for participation in study will be gotten.
- Confidentiality agreement will be signed by project implementation team and observed strictly.

Study design

This is a prospective intervention study with a qualitative component. Two study groups (Intervention and control groups will be selected. Intervention (which include participation in geriatric clinic and geriatric community health program and its benefits) will be provided in intervention groups. Pre and post study data will be analyzed to evaluate effect of intervention within the study group 12 months after intervention. Whereas control group with similar characteristics like intervention group in selected neighboring communities who do not receive the interventions will be assessed using same data collection tools after 12 months.

Firstly, Interviewer guided questionnaire will be used as a qualitative survey tool to assess baseline level of health status and access to health care (health facility, health workers, health examination and medication) among study participants and groups. Same tool will be used to assess health status and access to health care one-year post intervention.

Secondly, a standard checklist with quantitative weighting will be used to assess presence and functionality of community drug bank and community health program for older persons before intervention, and same checklist will be used to assess presence and functionality instituted programs in the study communities.

Thirdly, custom indicators will be used to monitor and evaluate effect of community drug bank and community health program on UHC and SDG3 among older persons respectively.

Effect of community drug bank on UHC will be evaluated through comparative analysis of cost of health care and medications before and after intervention, within and between study groups.

Effect of community health program on achieving SDG3 among older persons will be evaluated through comparative analysis of health status and vulnerability index of older persons before and after interventions within and between the study groups.

Therefore, for first quantitative analysis on effect of community drug bank, baseline data will be collected and compared on quantifiable out-of-pocket cost of health care which include health facility access, health workers' consultation and hospital admissions, 
Effect of Geriatric Program on Achieving Sustainable Development Goals and Universal Health Coverage among Older persons in Africa; A Nigeria Project Plan

health examination such as laboratory and medical investigation and medication costs. While for the second quantitative analysis on effect of community health program on achieving SDG3, data on health status indices and vulnerability index of participants, before and after interventions, will be collected, analyzed and compared within and between study groups.

\section{Sample size determination}

Out of 6632730 elderly persons aged $\geq 60$ in Nigeria [6], with sample size $\mathrm{n}$ and margin of error $\mathrm{E}$ given by $\mathrm{x}=\mathrm{Z}\left(\mathrm{c} /{ }_{100}\right)^{2} \mathrm{r}(100-\mathrm{r})$, $\left.\mathrm{n}=\mathrm{Nx}^{\mathrm{x}} /_{((\mathrm{N}-1) \mathrm{E}}^{2}+\mathrm{x}\right), \mathrm{E}=\operatorname{Sqrt}\left[{ }^{(\mathrm{N}-\mathrm{n}) \mathrm{x}} / \mathrm{n}(\mathrm{N}-1)\right]$, sample size of 385 is calculated, using Raosoft, (2004) sample size calculator for intervention study. Making allowance for $10 \%$ attrition rate, extra 39 participants will be added making a total of 424 minimum sample size.

\section{Sampling technique}

Multistage sampling technique will be used. By simple random sampling technique, 1 LGA each will be selected from the 3 states selected for study. Then by cluster and simple random sampling, 1 community each from list of communities in selected LGA will be selected. Using systematic random sampling, study population will be selected (with nth number, which will be calculated using population of community based project attendees by expected sample size per community). Out of each of the 3 communities, at least 142 persons per community will be selected to make up total sample size of 424 .

\section{Intervention}

The intervention will be a community-based household case management approach along with stakeholders' engagement and training of implementation team. There will be 5 intervention stages for the project;

- Stakeholders' engagement

- $\quad$ Project team engagement and training

- Identification, enrollment, assessment, and planning for beneficiaries using case management process approach

- $\quad$ Community based and health facility-based intervention as implementation/service provision approach for beneficiaries

- Data collection, monitoring and evaluation

\section{Stage 1 - Stakeholders' engagement}

Stakeholder and community gate keepers will be identified and stakeholders meeting held to discuss project and study aim/ processes. Stakeholders and gatekeepers will include researchers, state and local government ministry of health, primary health care development agency, pharmaceutical companies, organizations related to aging, community health workers, community health centers, community pharmacies and community leaders.

\section{Stage 2 - Project team engagement and training}

Community health team will be recruited and trained. The team will comprise of community case managers (community health volunteer), community pharmacy technician, laboratory technician and data/research assistants. Also, a linkage health facility/ hospital will be identified, and geriatric focal person selected from the facility -who will receive clients from community referrals and direct them appropriately to receive specialized hospital care as needed.

A standard training manual on geriatric care will be adapted to be used to train the community health workers' team and facility geriatric focal persons. Training will cover geriatric service provisions including assessment (health, psychosocial, safety and economic status) and intervention, referral, documentation, and data collection.

Specifically, the 7 steps of case management process for older persons will be understood which is foundation to implementation of the project. The 7 case management process include; 1) Identify; 2) Enroll; 3) Assess; 4) Plan; 5) Serve/implement; 6) Monitor; 7) Evaluate.

\section{GERIATRIC CASE MANAGEMENT PROCESS}

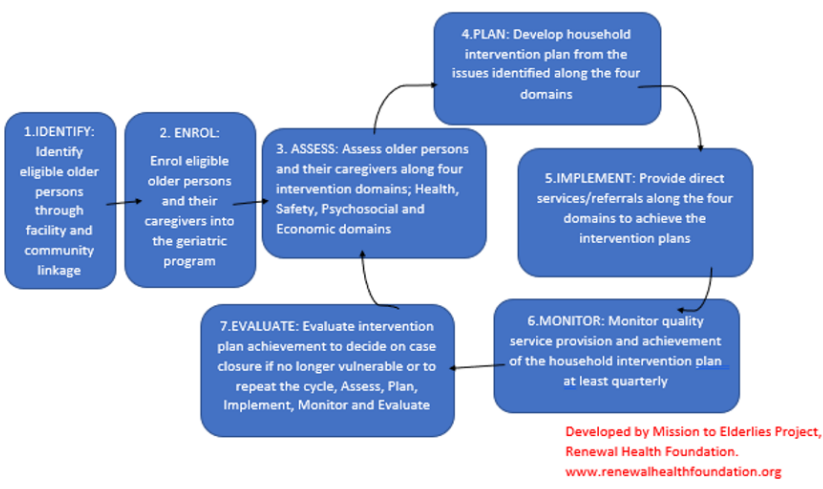

Figure 1: Conceptual framework for case management of community based health program among older persons. 
Stage 3 - Identification, enrollment, assessment and planning for beneficiaries using case management process approach

The first four parts of case management process- identify, enroll, assess and plan- will form the third stage of the project.

\section{Identification of beneficiaries}

Project beneficiaries who will also serve as study participants will be identified from community through two enrollment streams; community and health facility streams. While health facility stream will form older patients attending and receiving health care from health facilities in the community within the catchment areas, community streams will form older persons from the community who also meets the inclusion criteria.

\section{Facility stream identification}

To select beneficiaries from facility, patients records and registries will be assessed to identify those who meet inclusion criteria (older persons with reported age of 60 years, older person clinically diagnosed or confirmed with at least one chronic health condition, resident within study area as at the time of study, access to community health facility and community pharmacy).

\section{Community stream identification}

Beneficiaries will be selected from the community using two mediums; first is during 1 to 2 days community health intervention project and secondly is through community referral of eligible beneficiaries from community leaders.

\section{Enrollment of beneficiaries}

Enrollment of eligible beneficiaries as identified will be done both from facility and community.

\section{Facility enrollment}

The eligible beneficiaries (older persons with at least one documented chronic condition in the hospital record/folder) will be contacted by geriatric focal persons in the health facility to introduce the project aims and seek consent for enrolment. During patients' hospital visit, consent will be sort and signed and patient will be enrolled into the program through home visit. Enrollment via home visit will ensure patient is within catchment area and also enables enrollment of caregiver. The facility focal person has the responsibility of identifying the facility streams.

\section{Community enrollment}

Beneficiaries who are identified during community health projects or are referred by community leaders will be assessed during home visit. They will be referred to the partnering health center for medical examination and laboratory investigation for confirmation of at least one chronic medical condition which makes them eligible to be enrolled into the community drug bank project. At confirmation of chronic condition, they will be enrolled along with their caregiver.

In this case, the community health volunteers have responsibility of identifying community streams and for household enrollment of the beneficiaries. Household thus describes an older person (who is the index beneficiary) and the caregiver.

At the point of enrolment, enrollment form will be filled to collect sociodemographic data and other relevant inclusion requirement of the older person. Also, data from the caregiver of the index beneficiary will be collected. At enrollment, household folder will be opened for the household, containing forms for the index client (older person) and the caregivers). Other subsequent forms for assessment and service provision will be inputted into the folder.

\section{Assessment of beneficiaries}

After enrollment of eligible index clients and their caregivers, assessment form will be used to assess status of the older person. Assessment tool will be adapted from geriatric assessment tool and comprehensive geriatric assessment tools. \{Geriatric assessment tool\}. Each assessment question will be assigned quantitative numerical value. A presence of a condition will score 1 while its absence will score 0 . The quantitative values will be used to measure the vulnerability index of older person both at baseline and at post intervention. The project will group these assessments into four domains which will be used to categorize intervention domains. The four domains will include;

- Health domain (medical and health status)

- $\quad$ Safety domain (neglect, abuse and violence)

- Psychosocial domain (mental and psychological, social, spirituality and environmental and functional status)

- $\quad$ Economic domain (economic and dependency status) 
Domains for assessment of beneficiaries

Health domain

Health domain will assess medical/health status of the enrolled older persons.

Health assessment will cover questions that asses; medical condition like hypertension, diabetes, arthritis; fall risk; hearing and visual impairments; urinal incontinence; nutritional status; and effect of polypharmacy. Assessment/screening tool/form is used to identify patients who may need further comprehensive geriatric assessment or specific interventions. In addition, results from medical examination including laboratory investigation will be done as required and documented under the health domain assessment.

- Common Medical conditions: Common geriatric medical condition will be assessed through medical consultation, examination, laboratory investigation as required and documented under the health domain assessment. Example of which include; hypertension, diabetes, arthritis and heart diseases.

- Falls/Gait Disturbance: Tests of gait, balance, and functional reach help to assess patients' risk of falling. Underlying balance and gait disorders can best be detected by observing patients walking and performing balance maneuvers. To save time, this evaluation can be performed while the patient is entering or leaving the examining room. Assessment will include; including assessment of all medications, gait and balance, and orthostatic blood pressure readings, and vision testing. For patients who have tested positive for falls, a structured visit note can be used at follow-up visits

- Hearing Impairment: This involves asking patients whether they feel they have hearing impairment. An affirmative answer is considered a positive test for hearing loss, and patients should be referred to an audiologist. Another alternative is the whisper voice test, administered by whispering 3 to 6 random words at a set distance from the patient's ear and then asking the patient to repeat the words. Patients fail the screening if they are unable to repeat half of the words correctly. This should be done out of the patient's sight line to prevent lip reading, and the other ear should be covered.
However, the most accurate office test is the use of audioscope by specialists for which beneficiary can be referred for after positive screening tests.

- Visual Impairment: The Snellen eye chart is standard method of screening for visual impairment. This requires the patient to stand 20 feet from the chart and read letters, using corrective lenses. Patients fail the screening if. they are unable to read all of the letters on the 20/40 line with their eyeglasses on, and should then be referred for further evaluation by an ophthalmologist. Given the high prevalence of eye diseases in the older population and the potential for adverse health consequences of impaired vision, a visit with an optometrist or ophthalmologist is recommended every 1 or 2 years.

- Urinary incontinence: A simple and efficient screen for urinary incontinence is a 2-item questionnaire that can be administered by the provider: (1) "In the last year have you ever lost urine and gotten wet?" If so, (2) "Have you lost urine on 6 separate days?" Another single question, "Have you had urinary incontinence that is bothersome enough that you would like to know how it could be treated?" may convey additional value in helping to determine which patients would want further evaluation and therapy.

- $\quad$ Nutritional assessment: At the initial visit, patients should be weighed and asked about previous weight loss in the last 6 months. Height should also be obtained in order to calculate body mass index (BMI), which should be calculated at the initial visit and when there is a significant change in weight. There are also several self-administered screening instruments available. The Mini-Nutritional Assessment predicts adverse outcomes that may or may not be related to the nutritional components of the instrument. Nutrition assessment form will be used to assess nutrition status of older persons. Nutrition assessment will comprise of weight, BMI and food security assessment can be done in community and home visit.

- Polypharmacy: Adverse drug effects have been a challenge with orthodox medicine. For instance, some hypertensive drugs are associated with dizziness and headache. Much more in older person who take a handful of medicine due

Citation: Amara Frances Chizoba., et al. "Effect of Geriatric Program on Achieving Sustainable Development Goals and Universal Health Coverage among Older Persons in Africa; A Nigeria Project Plan". Acta Scientific Medical Sciences 5.12 (2021): 48-63. 
to comorbidities. Polypharmacy is very common in the older population, associated with adverse drug reactions, and can result in hospitalizations and increased morbidity. Patients often visit $>1$ healthcare provider and fill prescriptions at multiple pharmacies. Therefore, monitoring the many medications patients are on for drug-disease and drug-drug interactions is imperative at the initial and every follow-up visit. There are several ways to perform this task. Providers can ask their patients to bring all of their medications in to their visits with them, including over-the-counter medications. Previsit questionnaires can be completed prior to the initial visit with a complete list of patients' prescription and nonprescription medications. Office staff can then review these medication lists with patients and bring any discrepancies to the physician's attention at the time of the patient's visit.

\section{Safety domain}

Assessment for safety, abuse, and violence

This assesses the safety of older person relating to environment and home. It in turn assesses presence or risk of violence and elder abuse. Elder abuse suspicious index will be used to assess violence. Questions include; Within the past 12 months; Have you relied on people for any of the following: bathing, dressing, shopping, banking, or meals?; Has anyone prevented you from getting food, clothes, medication, glasses, hearing aids, or medical care, or from being with people you wanted to be with?; Have you been upset because someone talked to you in a way that made you feel shamed or threatened?; Has anyone tried to force you to sign papers or to use your money against your will?; Has anyone made you afraid, touched you in ways that you did not want, or hurt you physically?; Elder abuse may be associated with findings such as poor eye contact, withdrawn nature, malnourishment, hygiene issues, cuts, bruises, inappropriate clothing, or medication compliance issues. Did you notice any of these today or in the past 12 months? Outcome of assessment will be used for intervention such as education, caregiver's support and linkage to legal services as needed.

\section{Psychosocial domain}

The psychosocial domain will cover psychological, social, spirituality, sexuality/intimacy, functional and environmental assessment.
- Psychological assessment: Depression and other affective disorders are common in the older population The Patient Health Questionnaire-923 is a validated self-administered tool. A score of $>10$ has a sensitivity of $88 \%$ and a specificity of $88 \%$ for major depression. A 2-item version (PHQ-2) has been used for screening. This screener asks the patient, "Over the past 2 weeks, how often have you been bothered by any of the following problems? Little interest or pleasure in doing things. Feeling down, depressed, or hopeless." Responses are scored as follows: $0=$ not at all, $1=$ several days, $2=$ more than half the days, 3 = nearly every day. Persons who score $\geq 3$ have a $75 \%$ probability of having a depressive disorder. In patients who may not be able to complete a selfadministered tool (secondary to cognitive or visual impairment), the Geriatric Depression Scale may be more helpful.

- Social assessment: There is a great deal of interdependency between patients' social situations and their functional status. For example, persons dependent in ADLs or IADLs must have sufficient social or financial support to meet their needs. If patients are healthy but have no social support (family or friends), physicians should inquire who would help them should they have an increased level of need. For patients who are dependent in functional activities, it is important to ask who helps them perform each specific task (Figure 3; can be downloaded from http://www.geronet. ucla.edu/professionals/patientcare-resources). A variety of private and public resources can provide further assessment if the initial screening indicates a problem. Home assessments provided by home health social worker can also help further reveal levels of support at home.

- Functional and environmental assessment: Functional performance can be viewed as a measure of overall impact of health conditions in the context of a patient's environment and social support system. Therefore, it is essential to assess the patient's functional status at the initial visit, and any change in functional status should prompt further investigation. This can be assessed at 3 levels: basic activities of daily living (BADLs), instrumental activities of daily living (IADLs), and advanced activities of daily living (AADLs). The BADLs are the tasks that patients need to be able to complete on their own, or have assistance to complete, in order to be 
able to live in their own residences: transferring, toileting, bathing, dressing, continence, and feeding. The IADLS are the abilities one needs to maintain an independent household: going to market, driving or being able to use public transportation, use of phone, meal preparation, housework, home repair, laundry, taking medications, and handling finances. Patients may be dependent in $\geq 1$ IADLs but still able to live at home alone given they are independent in their ADLS and have family support to help pay bills, for example. Understanding the areas of impairment is essential to being able to meet the patient's needs with appropriate resources and also often helps with diagnosis. Measurement of AADLs (societal, family, recreational, and occupational tasks) can also be helpful in detecting changes in functional status prior to onset of disability. These questions can be completed prior to the initial visit with a use of a pre-visit questionnaire or can be formally asked at the initial visit.

- Cognitive assessment: Detection of cognitive impairments early can identify treatable conditions, such as ischemic brain disease, when risk factors can be then better controlled, helping to prevent progression of disease. The Mini-Mental State Examination, a 30-item interviewed administered assessment, is a validated and commonly used screening tool. There are also several shorter validated screens for cognitive impairment which include the Mini-Cog test, which combines 3-team registration and recall with clock drawing. These tools will be adapted for cognitive assessment to guide focused intervention.

- Spiritual assessment: Spirituality can have a significant influence on overall health. Though there are no formal assessment instruments that are common in clinical use, physicians should inquire whether spiritual or religious beliefs play an important role for patients. This can help give insights into their care plan and goals of care. In the hospitalized setting, chaplaincy support can be very helpful in helping to align patients' medical decisions with their belief system.

\section{Economic domain}

\section{Assessment of economic status}

The current economic crisis has affected many older persons, who are often on fixed incomes. Older persons who are dependent in their ADLs and/or IADLs, and do not have family readily available to fulfill these needs, will find it difficult paying for their needs or caregivers. Economics plays a large role in undernutrition, as well as ability to buy medications. Therefore, the assessment tool will probe on availability of social and economic support including health insurance coverage for older person. Assessment will include Households Economic Vulnerability Assessment (HEVA). Finding will be used to proffer older persons and their caregivers to benefit from cash transfer, income generating activities and free/ subsidized enrollment into National Health Insurance scheme.

\section{Planning for beneficiaries}

This will entail scoring of vulnerability index and development of care plan for intervention for beneficiaries (and households) assessed.

\section{Classification of beneficiaries by vulnerability index (VI) score}

Finding and scores from the assessment tool will be used to classify the beneficiaries based on vulnerability status. Each of the four domains will have three classification of vulnerability status which will inform the type and timeline of planning and intervention. Classification of vulnerability status include;

- Vulnerable; having a high vulnerability index (VI) score of $>70 \%$

- $\quad$ At risk of being vulnerable; having an average VI score of 40 to $60 \%$

- $\quad$ Not vulnerable; having a low VI score of $<40 \%$

\section{Care plan development for intervention}

This will involve a process of developing a written plan that details how to improve the well-being and safety of the older persons (index client) and the caregiver using finding from the assessment forms. The aim is to guide the community-based intervention tailored to need of the client which will in turn reduce vulnerability status where present and increase resilience.

Care plan form will be tailored into the four assessment domains which will be used to plan intervention in the four domains- health, safety, psychosocial, and economic domain. Care plan development enables planning of intervention for the beneficiaries assessed during household assessment by the community health worker. 
Intervention developed in care plan can be delivered through community program, home visit and referrals and linkages to health facility, national health insurance program, pension management organizations and agencies for right of the older persons.

Stage 4 - Community based and health facility-based intervention as implementation/service provision approach for beneficiaries

This will constitute of instituting geriatric clinics in health facilities, conducting mobile geriatric outreaches, establish community drug bank for beneficiaries and other community-based intervention programs like home visit by case managers, nutrition support, Household economic strengthening, safety and legal support, hosting beneficiaries forum to improve social interactions.

Therefore, after enrollment, assessment and planning for beneficiaries, the intervention approach will involve service provision. Enrolled beneficiaries will be clustered based on location and assigned to community case managers (community health workers) who manages a cohort of households.

The case manager conducts home visit on schedules or on demand and ensures appropriate referral as needed, reminds beneficiaries of health facility geriatric clinic appointments, mobilizes beneficiaries to attend mobile community geriatric outreaches, assists them to access medication at community drug bank, and is responsible for their care and services along the seven geriatric case management process.

\section{Geriatric clinic in community health facilities}

Working with health facility management, institute geriatric clinic in facility through training of facility works on geriatric program, provision of work tools, and referral directory for referrals as needed. As needed, budget can be developed for running of the geriatric clinic in the supported health facility.

Enrollment of beneficiaries into nation al health insurance scheme/programs

Working with health facilities, Health management organizations and National health insurance scheme (NHIS), beneficiaries will be enrolled into NHIS to reduce out of pocket expenses on health. While all beneficiaries will be sensitized on benefits of
NHIS, non-indigent beneficiaries will be guided on enrolment into NHIS while indigent beneficiaries will be enrolled freely or by subsiding enrollment fees. This will be supported through NHIS and through funding. Quality of service provisions through this scheme will be closely monitored by implementing partner.

\section{Mobile community geriatric outreach}

Monthly or quarterly community geriatric outreaches will be coordinated by community health team comprising of community case managers (community health worker), community pharmacy technician and laboratory technician and a supervising nurse/physician from linked facility.

Service provision at the community geriatric outreach will include assessment (across the four domains) using geriatric assessment form, medical and laboratory examinations, consultations, health education, nutrition support, prescription and administration of medications, documentation into forms, registers, and electronic medical record (EMR).

At end of community outreach, next appointment will be given for next outreach day which could be bi-monthly or quarterly. However, on advent of any condition needing intervention before the next appointment, the beneficiary or caregiver will contact their community case manager for assistance to supported health facility or other referrals as required.

\section{Geriatric drug bank}

Drug storage

In addition to the supported health facilities, community health pharmacies can be identified per community where drugs for geriatric health conditions will stored for pick up by older persons enrolled into the program. The community pharmacies will thus be the community geriatric drug bank where drugs are stored for end users to access based on prescription.

\section{Supply chain management}

The project will engage a supply chain and logistic officer to procure or receive drugs and other commodities from manufacturing/supplying company or pharmacy to the community pharmacy (geriatric drug bank). Commodity request and requisition form will be used by supply chain officer and facility/community phar- 
macist to request for medications as needed and for requisition of exhausted supply. This ensures steady and consistent supply of needed medications.

Home base care by case managers

Community case managers assigned to older persons enrolled in the program will be responsible for providing home based care for their beneficiaries at home on scheduled dates or as need arises. Home visit will be scheduled at least once a quarter for all beneficiaries and monthly for beneficiaries who has $>70 \%$ in vulnerability index score. Services will include assessment and intervention across the four domains, physical examination, health education, medication support, nutrition support and safety support. Referrals will be made to supporting facility and emerging issues reported to implementing partner (or the community-based organization managing the project) for prompt intervention. Service provision during home visit will be documented in data capturing tools and peculiar findings discussed during case management review meetings.

\section{Nutrition intervention}

Nutrition assessment form will be used to assess nutrition status of older persons during community clinic and home visit. A beneficiary that shows nutritional vulnerability will be planned and supported for nutrition intervention. Nutrition intervention will include provision of MEFOOD (Packed nutrition boost meal comprising of soyabeans, groundnut, crayfish and unripe plantain). Other intervention will include food provision through food bank, home gardening, nutrition counselling, food demonstration and linkage to health facility.

Household economic strengthening

Beneficiaries and caregivers who during household economic vulnerability assessment had poor scores under economic domain will be further assessed for household economic strengthening (HES) program. HES programs will include cash transfer, income generating activities, financial education and vocational skill acquisition, village loan savings association and linkage to beneficial loan access for caregivers.

\section{Caregivers/beneficiaries forum}

Caregivers' forum will be held for caregivers of beneficiaries (and beneficiaries) where education on care of older persons will be taught using standard curriculum. This will be done in batches in bi-monthly and quarterly.

\section{Referral services}

Referral for services not provided at community geriatric clinic, drug bank and home base care will be referred to appropriate institutions. Matter relating to health domain will be referred to partnering health center, matters relating to safety, psychosocial and economic domains will be referred to agencies for rights of older persons, psychosocial experts, and financial institutions respectively. The referral services will be overseen by the implementing partner or civil society organization implementing the project.

\section{Stage 5 - Data collection and monitoring and evaluation}

Assessment and service provision forms and registers, care plan and monitoring checklist will be used to document assessment finding, service provision, care plan for intervention and outcome of monitoring respectively. Data information management system will be developed and used to capture electronically the project activities which will be used for data collation, analysis and presentation.

\section{Data collection}

To answer the first research question (What is the health status and level of access to health care among older persons in study group), data collection will be done using interviewer guided questionnaire as a qualitative survey tool to assess baseline level of health status and access to health care (health facility, health workers, health examination and medication) among study participants. Same tool will be used to assess any changes in health status and access to health care.

To answer the second research question (Is community drug banks and community health program for older persons present, feasible and functional), data collection will be done using a standard checklist with quantitative weighting. This will be used to assess presence and functionality (if present) of community drug bank and community health program for older persons before intervention and same will be used to assess presence, and functionality of the instituted program 12 months after intervention in communities where study groups are.

To answer the third and fourth research questions, (What is the effect of community programs \{NHIS and community drug bank\} 
on UHC among older persons? And "is there effect of community health program \{geriatric clinics, mobile outreaches, home based care by case managers, education and service provisions along the four intervention domains\} on achieving SDG (3) among older persons?"), data will be collected using data capturing tools (DCT). For the third research question, DCT/checklist will be tailored to assess out-of-pocket cost of health care (health facility access, health workers' consultation and hospital admissions, health examination such as laboratory and medical investigation and medication costs). And for the fourth research question, questions will be tailored to assess health status and vulnerability index before and 12 months after intervention.

Data from assessment and service provisions updated on DCTs will be entered electronically into Geriatric Data Management Information System (GDMIS) by data entrants and evaluated by research officers. Custom indicators will be used to monitor and evaluate effect of community health program on UHC and SDG3 among older persons.

Effect of community drug bank and NHIS on UHC will be evaluated through comparative analysis of out-of-pocket cost of health care and medications before and after intervention. On the other hand, effect of community health program on achieving SDG3 among older persons will be evaluated through comparative analysis of health status and vulnerability index of older persons before and after interventions within and between the study groups.

\section{Monitoring and evaluation}

Monthly monitoring visit will be conducted by project management team to the supported health facilities, community pharmacies, mobile geriatric outreach groups and caregivers/beneficiaries' forum. Spot checks to beneficiaries' households will also be done to monitor quality of service provisions. Quarterly review meeting will be held by principal investigators, project management team and community health teams to monitor achievement against indicator targets. Site Monitoring System (SIMS) checklist and Data quality Assurance (DQA) checklist will be used to monitor progress and quality of project implementation, outcome of which will be used for quality assurance, assessment, and intervention. Thus, data collection and analysis will be done using monthly data collection forms to monitor effect of intervention while annual data will be used to evaluate effect at one-year post intervention.
Data analysis

Data will be analyzed using descriptive analysis for descriptive variables such as age, sex, location, income, occupation, and disease condition. Frequency distribution will be used to analyze responses from questionnaire. And SPSS version 21 will be used for analysis of variables.

Each question in checklist with positive response will be assigned 1 and negative response assigned 0 . Percentage scores will be calculated for each of the assessment domains. Percentage scores will be compared between baseline and post intervention assessments. T-test, chi square and ANOVA will be used to analyze and compare effects of intervention, before and after interventions within and between the intervention and control groups. Significance will be described as $\mathrm{p}<0.01$ at $95 \% \mathrm{CI}$ and $5 \%$ error.

\section{Results and Discussion}

Results will be published at different stages of implementation of the project.

\section{Conclusion}

Review of literatures and from anecdotal data shows that there is gap in planning of health care for older persons whose population is increasing exponentially. This project proposal aims to implement and evaluate effect of innovative community health program on achieving SDGs and UHC goals among older persons in Nigeria. A finding that is aimed to be disseminated for adaption and adoption for geriatric programing in the African context.

\section{Bibliography}

1. WHO. "Healthy Ageing and the Sustainable Development Goals".

2. United Nations. "World population aging" (2017).

3. WHO- "World report on Ageing and Health".

4. Ba Omotara., et al. "Assessment of the Determinants of Healthy Ageing among the Rural Elderly of North-Eastern Nigeria" (2012).

5. United Nations. "World Population aging" (2015).

6. Central Intelligence Agency. Africa: Nigeria, The World Factbook (2019). 
7. Federal ministry of Health. Nigerian Demographic and Health Survey (NDHS) (2018).

8. WHO. World report on population and aging (2015).

9. Morgan Clark., et al. "Innovative Public-Private Models to Enhance Aging in Place in the United States" (2016).

10. WHO. "Universal Health Coverage and Ageing".

11. Ritu Sadana., et al. "Universal health coverage must include older people". Bulletin of the World Health Organization 96 (2018): 2-2A.

12. Araujo de Carvalho I., et al. "Organizing integrated health-care services to meet older people's needs". Bulletin of the World Health Organization 95.11 (2017): 756-763.

13. Luís Midão., et al. "Adherence to medication in older adults" (2017).

14. Christine Tassone Kovner., et al. "Who Cares for Older Adults?" Workforce Implications of an Aging Society.

15. SL Rosen and DB Reuben. "Geriatric assessment tools" (2011).

16. WHO improving accessibility of medications (Access to medicines: making market forces serve the poor).

17. Medicare. "Helping the Elderly Pay for Prescription Drugs" (2019).

18. New York department of health. "Elderly Pharmaceutical Insurance Coverage (EPIC) Program" (2015).

19. Amara Frances Chizoba., et al. "Accessibility of Medications by Chronically Ill Older Patients: A Cross-Sectional Assessment of Universal Health Coverage Among the Elderly in Nigeria". Acta Scientific Medical Sciences 4.2 (2020): 01-09.

20. Eugenia L Siegler., et al. "Community-Based Supports and Services for Older Adults: A Primer for Clinicians".

21. Mission to Elderlies Project, Renewal Health Foundation (2021).

Volume 5 Issue 12 December 2021

(C) All rights are reserved by Amara Frances Chizoba., et al. 